\title{
PCR-Based Genomic Deletion Analysis of RD-Regions in the Identification of Mycobacteria Isolated from Adverse Events Following BCG Vaccination or TB Suspected Cases
}

\author{
KATARZYNA KRYSZTOPA-GRZYBOWSKAㄹ, SYLWIA BRZEZIŃSKA², EWA AUGUSTYNOWICZ-KOPEĆ², \\ EWA AUGUSTYNOWICZ ${ }^{1}$ and ANNA LUTYNSSKA ${ }^{1}$ \\ ${ }^{1}$ National Institute of Public Health - National Institute of Hygiene, Warsaw, Poland \\ ${ }^{2}$ National Tuberculosis and Lung Diseases Research Institute, Warsaw, Poland
}

Submitted 9 August 2013, revised 5 February 2014, accepted 4 July 2014

\begin{abstract}
Early identification of mycobacterial species is crucial for early diagnosis. PCR-multiplex method performed on randomly chosen 54 mycobacteria isolates originating from clinical samples was found to be an inexpensive, quick and reliable alternative for commercially available diagnostics tests. Although the results of gene probes identification performed by NTLDR were generally consistent with multiplex PCR, two mixed Mycobacterium bovis BCG/Mycobacterium tuberculosis infections and a single misdiagnosis of M. tuberculosis with M. bovis were found. The routine application of multiplex-PCR has the potential to make diagnostics surveillance studies feasible.
\end{abstract}

Key words: Mycobacterium tuberculosis complex, Mycobacterium bovis BCG, Mycobacterium bovis, adverse events following BCG immunizations, multiplex PCR

In spite of many research and development investments, tuberculosis (TB) caused by the bacillus Mycobacterium tuberculosis is still a main prevalent infectious disease and worldwide human killer. In 2010, 8.8 million incident cases of TB were estimated to appear globally. The elimination of tuberculosis by 2050 is the prioritized long-term goal of the World Health Organization (WHO). However, its success depends on the effectiveness of strategies undertaken for the improvement of current diagnostics, treatment, vaccination and new tools to control and fight the epidemics as well (WHO, 2012). Although advanced and more efficacious vaccine is highly expected, attenuated M. bovis Bacillus Calmette-Guérin (BCG) is still the only available one against TB, included in the WHO Expanded Program of Immunization since 1974 (WHO, 2004). BCG vaccine protects against severe forms of tuberculosis in newborn children, however against pulmonary TB in adults heterogeneously (Kaufmann et al., 2010).

The most common method for diagnosing TB worldwide is the developed more than 100 years ago sputum smear microscopy. In more developed laboratory, cases of TB may also be diagnosed via gold standard culture methods or, increasingly, using rapid molecular tests (WHO, 2012). An inexpensive acidfast staining and microscopy, because of rather low sensitivity, provide no information on the species of mycobacteria causing disease and are relatively insensitive and not useful to identify paucibacillary or extrapulmonary TB. Conventional laboratory culture and chemical testing procedures are regarded as timeconsuming and not practical for surveillance purposes (Warren et al., 2006).

Problems with species identity within the M. tuberculosis complex originate from high DNA-DNA homology and identical 16S rRNA sequences (Kasai et al., 2000). This group consists of nine closely related species: M. tuberculosis, Mycobacterium microti, M. bovis, M. bovis Bacille Calmette-Guérin (BCG), Mycobacterium canetti, Mycobacterium caprae, Mycobacterium mungi and Mycobacterium pinnipedii (KrysztopaGrzybowska et al., 2012). The high degree of sequence conservation among the members of the M. tuberculosis complex results in difficulties in routine diagnostics in different world parts. Precise species diagnosis is of great importance for the treatment of individual patients and epidemiological purposes as well, especially in areas where tuberculosis has reached epidemic

\footnotetext{
* Corresponding author: A. Lutyńska, National Institute of Public Health - National Institute of Hygiene, 24 Chocimska Street, 00-791 Warsaw, Poland; phone: +48 225421 213; fax: +48 225421 311; e-mail: alutynska@pzh.gov.pl
} 
proportions or wherever the transmission of i.a. M. bovis between animals or animal products and humans is a problem. Because of the species range able to induce the infection in humans, improved diagnostics is also highly expected to rapidly identify all species of M. tuberculosis complex, including M. bovis BCG, recovered from immunocompromised patients (Parsons et al., 2002).

In our previous study we evaluated the capacities of a few described previously nucleic acid amplification techniques to identify M. tuberculosis complex representatives (Krysztopa-Grzybowska et al., 2012). As the PCR-based method described by Warrenet et al. (Warren et al., 2006) was found to be the most useful, the current study involved the retrospective verification of the precision of the species identity performed on mycobacteria grown from individuals suspected of adverse events following BCG immunization (AEFI) or M. tuberculosis infection collected at the National Tuberculosis and Lung Diseases Research Institute (NTLDRI) in Poland comparing to appropriate reference strains. The goal of the study was to reevaluate the precision of the identification of mycobacteria for purposes of its diagnostics improvement.

A total of 54 mycobacterial strains isolated from 30 individuals suspected of adverse events following BCG vaccination and $24 \mathrm{~TB}$ patients collected at the NTLDRI between 2007-2012, were included in the study. 35 mycobacteria were initially identified as M.tuberculosis by the niacin test, the use of gene probes (AccuProbe; GenProbe, San Diego, CA), and mycolic acid analysis by high-pressure liquid chromatography in NTLDRI. All strains were grown on solid Löwenstein-Jensen medium for about 20 days at $37^{\circ} \mathrm{C}$, harvested, heat inactivated and coded. The following reference strains were used: M.bovis BCG Moreau originating from BCG vaccine lot no. 00108, M. bovis BCG Danish 1331 (NIBSC, 07/270), M. bovis BCG Tokyo 172 (NIBSC, 07/272), M.tuberculosis H37Rv (ATCC 25618), M. bovis (ATCC 19211), M. microti (ATCC 19422), Mycobacterium africanum
(ATCC 25420), M. pinnipedii (ATCC BAA-688), and M. canetti (kindly obtained from Prof. Dr. Dick van Soolingen from Tuberculosis Reference Laboratory, National Institute for Public Health and the Environment, The Netherlands).

Chromosomal DNA was isolated from mycobacterial strains as described previously (van Soolingen et al., 1991). In the study we performed PCR-based genomic deletion analysis involved PCR method with use of primers specific for RD1 (RD1F AAG CGG TTG CCG CCG ACC GAC C; RD1R GAG GCG ATC TGG CGG TTT GGG G; RD1 CTG GCT ATA TTC CTG GGC CCG G), RD4 (RD4F ATG TGC GAG CTG AGC GAT G; RD4R AAA GGA GCA CCA TCG TCC AC; RD4 TGT ACT ATG CTG ACC CAT GCG), RD9 (RD9F CAA GTT GCC GTT TCG AGC C; RD9R GCT ACC CTC GAC CAA GTG TT; RD9 CAA TGT TTG TTG CGC TGC), and RD12 (RD12F GTG TTG CGG GAA TTA CTC GG; RD12R AGC AGG AGC GGT TGG ATA TTC; RD12 GGG AGC CCA GCA TTT ACC TC) regions (Warren et al., 2006) as in our previous study (Krysztopa-Grzybowska et al., 2012) it was found capable of precisely and simultaneously differentiating virulent and avirulent mycobacteria or virulent species of M. tuberculosis complex. Each PCR reaction was carried out in a volume of $50 \mu \mathrm{l}$ with $5 \mu \mathrm{l}$ of template (approximately 50 ng of DNA), $1 \times$ PCR buffer (SIGMA), $1.5 \mathrm{mM} \mathrm{MgCl}_{2}$ (SIGMA), $0.2 \mathrm{mM} \mathrm{dNTP}$ mix (Promega), $0.2 \mu \mathrm{M}$ of RD1F, RD1R, RD2F, RD2R, RD4F, RD4R, RD12F, RD12R primers, $0.4 \mu \mathrm{M}$ of RD1, RD2, RD4, RD9F, RD9R, RD12 primers, $0.8 \mu \mathrm{M}$ of RD9 primer and 2.5 U Taq DNA polymerase (SIGMA). The thermal conditions were set as an $94^{\circ} \mathrm{C}$ initial denaturation ( $10 \mathrm{~min}$ ), followed by 30 cycles of $94^{\circ} \mathrm{C}$ denaturation $(1 \mathrm{~min}), 55^{\circ} \mathrm{C}$ annealing $(1 \mathrm{~min}), 72^{\circ} \mathrm{C}$ extension $(2 \mathrm{~min})$ and $72^{\circ} \mathrm{C}$ final extension $(10 \mathrm{~min})$. The PCR products were analyzed by electrophoresis on $3 \%$ $(\mathrm{w} / \mathrm{v})$ agarose gel containing Midori Green with $100 \mathrm{bp}$ molecular marker.

Among 54 mycobacteria isolates, 19 were easily identified as M. bovis BCG (Fig. 1A.) as they showed
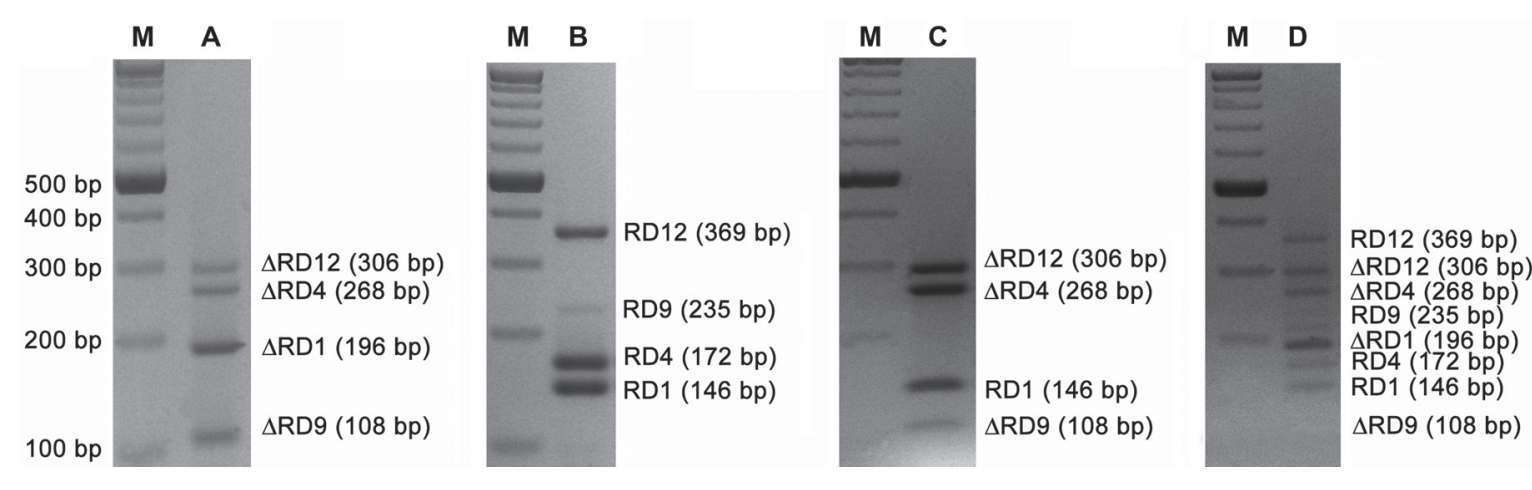

Fig. 1. Multiplex PCR of clinical strains: A. 19 clinical strains, B. 32 clinical strains, C. 1 clinical strain D. 2 clinical strains; $\mathrm{M}$, molecular marker $100 \mathrm{bp}$. 
Fig. 2. Multiplex PCR of references strains: lane 1, M. bovis BCG Moreau; lane 2, M. bovis BCG Danish; lane 3, M. bovis BCG Tokyo; lane 4, M. tuberculosis H37Rv; lane 5, M.bovis; lane 6, M. africanum; lane 7, M. microti; lane 8, M. canetti; lane 9, M. pinnipedii; lane 10, negative control; M, molecular marker $100 \mathrm{bp}$.

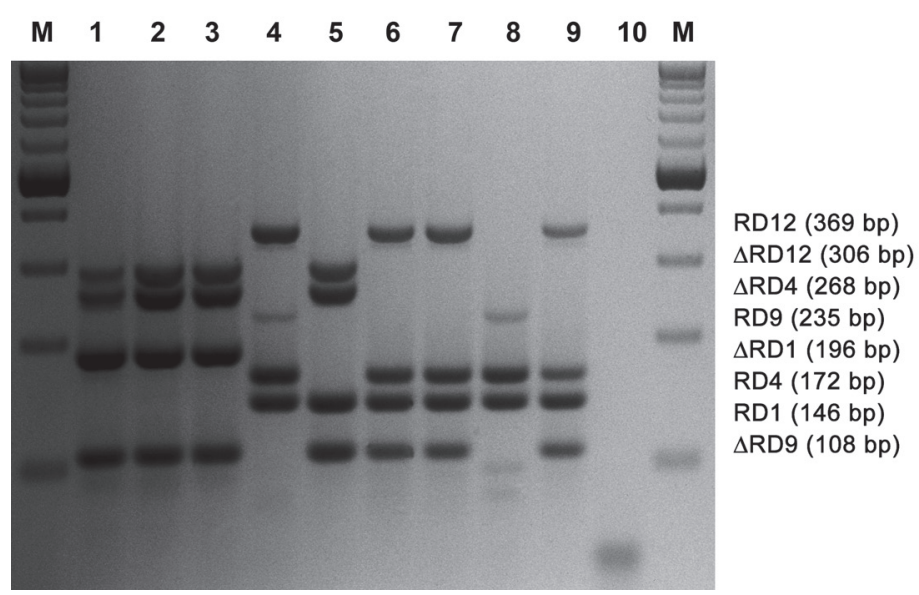

a multiplex PCR profiles presence of $108 \mathrm{bp}(\triangle \mathrm{RD} 9)$, $196 \mathrm{bp}(\Delta \mathrm{RD} 1), 268 \mathrm{bp}(\Delta \mathrm{RD} 4)$ and $306 \mathrm{bp}(\Delta \mathrm{RD} 12)$ amplified fragments identical as profiles of vaccine substrains - M. bovis BCG Moreau, M. bovis BCG Danish and M. bovis BCG Tokyo (Fig. 2). Thirty two other mycobacteria isolates presented the same profiles as those of M. tuberculosis reference strains (Fig. 1B, Fig. 2) with fragments of $146 \mathrm{bp}$ (RD1), $172 \mathrm{bp}$ (RD4), $235 \mathrm{bp}$ (RD9) and $369 \mathrm{bp}$ (RD12). Only one isolate was identified as M. bovis - $108 \mathrm{bp}(\Delta \mathrm{RD} 9), 146 \mathrm{bp}(\mathrm{RD} 1), 268 \mathrm{bp}$ $(\triangle \mathrm{RD} 4)$ and $306 \mathrm{bp}(\triangle \mathrm{RD} 12)$ (Fig.1C). Two specimens showed co-infection with M. tuberculosis and M. bovis BCG (Fig. 1D).

Differentiation of M. tuberculosis complex species is important for the accurate diagnosis of mycobacterial disease, public health surveillance and appropriate case management particularly in adult and pediatric patients with human immunodeficiency virus (HIV) infections or other related to immune suppression. In most countries, there are only limited data on the total incidence or prevalence of mycobacterial disease due to specific complex members, which is partly due to the absence of simple tests able routinely possible to apply (Warren et al., 2006). In Poland a network of bacillus laboratories dealing exclusively with diagnosis in tuberculosis suspected patients is established. In 2010 there were as many as 90 active bacillus laboratories registered. Only a few of them had a sufficient panel of diagnostics tests available but all were able to perform microscopic examination and classic culture, and some - to identify main species of mycobacteria, determine the drug sensitivity or perform basic genetic testing (Augustynowicz-Kopeć and Zwolska, 2010). Most of the laboratories however, were unable to quickly differentiate between M. bovis BCG and other members of $M$. tuberculosis complex, implying the possibility of development of BCG complications which may be considered and treated as tuberculosis. Because of difficulties in the proper identification of M. bovis BCG substrains isolated from the patients, the prevalence of BCG vaccine-induced infections is not exactly known. Knowledge of M. bovis BCG infection would be of particular interest to the clinician responsible for the therapy (Zwolska et al., 2004). Although gene probes and mycolic acid analysis by high-pressure liquid chromatography is routinely used for confirmation of virulence in the mycobacteria grown from the patients in NTLDRI, the application of a simple and fast molecular tool capable to distinguish the virulent species of the M.tuberculosis complex is lacking, as commercially available genetics tests are expensive. To increase the sensitivity and specificity of molecular diagnostics, multiplex-PCR dealing with genomic deletion analysis of the RD-regions described previously, was evaluated and validated (Warren et al., 2006; Krysztopa-Grzybowska et al., 2012) and then used to verify the previous results of identity by retrospective study of randomly chosen mycobacteria cultures grown from suspects of AEFI from BCG vaccination and suspected of tuberculosis. Among 35 mycobacteria cultures primarily identified at the NTLDRI as virulent mycobacteria, a validated multiplex-PCR allowed to find two cultures as mixture M. bovis BCG with M. tuberculosis, as RD12, $\Delta \mathrm{RD} 12, \Delta \mathrm{RD} 4, \mathrm{RD}$, $\triangle \mathrm{RD} 1, \mathrm{RD} 4, \mathrm{RD} 1$ and $\triangle \mathrm{RD} 9$ fragments were easily identified. As mixed infections were described (Warren et al., 2006), the success of their detection is dependent on the ratio of both species presence in the sample. Because of the rare character of mixed infections, detection systems available or being in use routinely might be not sensitive enough because of difficulties with the validation.

One isolate was found to be misdiagnosed as M. tuberculosis instead of M. bovis. M. bovis is the etiological agent of tuberculosis in many species of animals causing worldwide annual losses to agriculture of $\$ 3$ billion, but the human burden of tuberculosis caused by the bovine tubercle bacillus is still unknown (Garnier et al., 2003; de Kantor et al., 2008). As reports on M. bovis zoonosis are described, there is a need to 
undertake the task to differentiate human infections and those caused by other mycobacteria of the complex which not necessarily lead to clinical disease. Noncommercial methods, e.g. spoligotyping or REA with the IS6110 probe were suggested as more appropriate (de la Rua-Domenech, 2006), as commercial amplification methods and DNA probes can be insufficiently discriminatory for these two species. The low M. bovis infection cases in humans despite high animal reservoir, however, provide no evidence of recent human to human or animal to human transmissions in London and Southeast England (Stone et al., 2011). In The Netherlands, however a recent report on M. bovis infected young resident health professional female was found however untraced and different from cases previously found mainly among $>60$ years old suffering from reactivations or younger immigrants (Akkerman et al., 2012). Misdiagnosis of M. tuberculosis with M. bovis is possible but the treatment is very similar and that species determination in light of clinical outcome is less important than for surveillance purposes.

The described multiplex PCR analysis has been routinely applied since the study presented here as an easy and quick alternative or confirmation tool to identify the species of the complex in isolates grown from patients with tuberculosis and suspected of AEFI after $B C G$ vaccination. The method described is significantly faster and simpler than conventional ones taking generally weeks to achieve the same level of classification. Also its simplicity, low workload and especially low costs are favorable when compared with spoligotyping, REA with the IS6110 probe or Genotype MTBC (Hain Lifescience $\mathrm{GmbH}$ ) commercially available alternatives. The distinguishing between species causing the disease in humans and animals belonging to the M. tuberculosis complex and the ability to detect mixed infections is extremely important both for diagnostic and epidemiological studies purposes. Wider validation studies for mixed infection detection with the application of commercially available diagnostics tools or home-made developed methods are expected in the future.

Conflict of interest statement: the authors of this manuscript do not have a commercial or other association that might pose a conflict of interest and the study was not funded by any company.

\section{Acknowledgments}

This work was done in National Institute of Public Health - National Institute of Hygiene, Warsaw, Poland.

This study was supported by a grant for scientific research (NN404 207 239) from the Ministry of Science and Higher Education in Poland.

\section{Literature}

Akkerman O., K. Loo, D. Nijmeijer, T. Werf, B. Mulder, K. Kremer, D. Soolingen and A. Zanden. 2012. Mycobacterium bovis infection in a young Dutch adult: transmission from an elderly human source? Med. Microbiol. Immunol. 201:397-400.

Augustynowicz-Kopeć E. and Z. Zwolska. 2010. Progress in diagnosis and epidemiology of Mycobacterium tuberculosis (in Polish). Post. Mikrobiol. 49: 151-156.

Garnier T., K. Eiglmeier, J.-C. Camus, N. Medina, H. Mansoor, M. Pryor, S. Duthoy, S. Grondin, C. Lacroix, C. Monsempe and others. 2003. The complete genome sequence of Mycobacterium bovis. Proc. Natl. Acad. Sci. USA 100: 7877-7882.

De Kantor I.N., M. Ambroggi, S. Poggi, N. Morcillo, M.A. Da Silva Telles, M. Osório Ribeiro, M.C. Garzón Torres, C. Llerena Polo, W. Ribón, V. García and others. 2008. Human Mycobacterium bovis infection in ten Latin American countries. Tuberc. Edinb. Scotl. 88: 358-365.

Kasai H., T. Ezaki and S. Harayama. 2000. Differentiation of phylogenetically related slowly growing mycobacteria by their gyrB sequences. J. Clin. Microbiol. 38: 301-308.

Kaufmann S.H.E., G. Hussey and P.-H. Lambert. 2010. New vaccines for tuberculosis. Lancet 375: 2110-2119.

Krysztopa-Grzybowska K., S. Brzezińska, E. AugustynowiczKopeć, Z. Zwolska, E. Augustynowicz and A. Lutyńska. 2012. The capacity of Mycobacterium tuberculosis complex species and M. bovis BCG substrains specific identification-implications for optimized PCR-based diagnostics in adverse events following vaccination suspected cases. Pol. J. Microbiol. 61: 299-303.

Parsons L.M., R. Brosch, S. T. Cole, A. Somoskövi, A. Loder, G. Bretzel, D. Van Soolingen, Y. M. Hale and M. Salfinger. 2002. Rapid and simple approach for identification of Mycobacterium tuberculosis complex isolates by PCR-based genomic deletion analysis. J. Clin. Microbiol. 40: 2339-2345.

De la Rua-Domenech R. 2006. Human Mycobacterium bovis infection in the United Kingdom: Incidence, risks, control measures and review of the zoonotic aspects of bovine tuberculosis. Tuberc. Edinb. Scotl. 86: 77-109.

Van Soolingen D., P.W. Hermans, P.E. de Haas, D.R. Soll and J.D. van Embden. 1991. Occurrence and stability of insertion sequences in Mycobacterium tuberculosis complex strains: evaluation of an insertion sequence-dependent DNA polymorphism as a tool in the epidemiology of tuberculosis. J. Clin. Microbiol. 29: 2578-2586. Stone M.J., T.J. Brown and F.A. Drobniewski. 2011. Human Mycobacterium bovis Infections in London and Southeast England. J. Clin. Microbiol. 50: 164-165.

Warren R.M., N.C. Gey van Pittius, M. Barnard, A. Hesseling, E. Engelke, M. de Kock, M.C. Gutierrez, G.K. Chege, T.C. Victor, E.G. Hoal and others. 2006. Differentiation of Mycobacterium tuberculosis complex by PCR amplification of genomic regions of difference. Int. J. Tuberc. Lung Dis. 10: 818-822.

World Health Organization. 2004. Weekly epidemiological record. 79: 27-38.

World Health Organization. 2012. Global tuberculosis report 2012. World Health Organization, Geneva. http://apps.who.int/iris/bitstr eam/10665/75938/1/9789241564502_eng.pdf

Zwolska Z., E. Augustynowicz-Kopeć, A. Zabost, J. Ziółkowski, J. Buchwald, M. Płończak, W. Walas and M. Ziebiński. 2004. Modern microbiological methods in diagnosis of adverse reactions after BCG vaccination. Case reports (in Polish). Pneumonol. Alergol. Pol. 72: 505-511. 